\title{
Construction and Application of Economic Management Fuzzy Decision Model Based on Fuzzy Relevance Method
}

\author{
Zhaoying Ouyang $(\mathbb{D}$ \\ School of Marxism, Shanghai Lixin University of Accounting and Finance, Shanghai 201209, China \\ Correspondence should be addressed to Zhaoying Ouyang; 20119003@lixin.edu.cn
}

Received 24 November 2021; Accepted 27 December 2021; Published 20 January 2022

Academic Editor: Miaochao Chen

Copyright (c) 2022 Zhaoying Ouyang. This is an open access article distributed under the Creative Commons Attribution License, which permits unrestricted use, distribution, and reproduction in any medium, provided the original work is properly cited.

\begin{abstract}
Fuzzy mathematics has been used more and more extensively and deeply in various fields since its emergence, and it has been more and more recognized. However, there is less research on fuzzy mathematics in the field of economic management. This paper attempts to introduce fuzzy theories and methods into economic management. On the premise of introducing fuzzy decisionmaking theories and methods, several fuzzy theories and methods that are commonly used in economic management are given. The fuzzy correlation method of economic phenomena is proposed, and the method is used to study the correlation between economic phenomena. Besides, this paper also uses fuzzy methods to analyze the relationship between economic phenomena-fuzzy relevance, and uses fuzzy two-way decision-making methods to carry out economic management and decision-making so that the decision-making results are more reasonable, scientific, and operability. In addition, this paper rationally explains the phenomena and laws existing in economic activities from a fuzzy perspective and gives models for fuzzy inference, diagnosis, and production decision-making, which have important meaning and profound theoretical value and practical value.
\end{abstract}

\section{Introduction}

One of the latest application fields of fuzzy set theory is social science, especially in the field of economic management. The fruitful results of traditional mathematics in economic management should be attributed to the following reasons. First, these theories are derived from classical mathematics, based on classical set theory, double-valued logic, and arithmetic operations. This is unrealistic in economic life for two reasons. (1) Human natural language reasoning and decision-making play an important role in economic phenomena, and natural language is inherently uncertain. Classical mathematics cannot express this type of uncertainty. (2) It is related to the complexity of the model. With economic development, many related phenomena occur, which requires more complex mathematical models. Because the model that represents the real world is too complex, only simple methods can be used. However, a lot of meaningful content and data will be lost in the process of simplification. When a simplified model is used to predict certain and accurate results, the application range of the model is reduced, which makes the prediction result less relevant to the real world. Only when the model contains uncertainty can this correlation be preserved. That is to say, within the limits of acceptable complexity, accurate and deterministic results can be obtained, and the correlation between them is also preserved [1-5].

The fuzzy theory is applied to the field of economic management, and some methods such as fuzzy preference theory, fuzzy game theory, fuzzy production management, and fuzzy decision-making methods have gradually emerged [6-8].

The most successful application field of fuzzy set theory is fuzzy control. Various control clamors vary in many ways according to the different problems to be solved. For example, the most complex control is typically like a robot, requiring coordinated actions in a multidimensional space, while the simplest control only requires maintaining the existing state of a single variable [9-12].

The traditional controller is constructed according to the mathematical model of the control process, and the area fuzzy controller is mainly derived from the human operation. This knowledge comes from fuzzy control rules. Each rule is derived from a conditional fuzzy theorem [13-16]. For 
example, if the speed is equally (negative) low (about the expected speed) and the speed change is equally (negative) high, then the power must be equally (positive) high.

Here, the speed and speed change are the variables that can be observed during the control process, and the power represents the variables of the controller action [17-21]. The language value is equivalently (negative) low, equivalently (negative) high, and equivalently (positive) high which represents the corresponding fuzzy number.

The number of fuzzy controller series of different complexities that have achieved obvious results is quite huge. One of the series is the use of simple fuzzy controllers in many consumer products: washing machines, vacuum cleaners, electric shavers, dishwashers, electric cookers, video recorders, cars (antislip brakes, automatic steering, speed control, and other functions), refrigerator humidifier, and air conditioner. More complex fuzzy controllers are mainly used in group control systems, such as subways, urban traffic, and different industrial control systems. The most complex fuzzy controller that has been successfully verified and implemented is to control the helicopter's flight by transmitting natural language commands to the helicopter through the ground wireless transmitter. In short, this achievement is far beyond the scope of traditional control theory. Of course, the fuzzy controller combined with traditional control theory will achieve greater success. Fuzzy controllers are often combined with neural networks $[22,23]$. The static learning ability of the latter makes the fuzzy controllers better adapt to various external environments (conditions).

Another area of successful application of fuzzy set theory is the wide application of decision support. In fact, fuzzy theory has penetrated into all kinds of decision support, such as multiobjective, multiperson, or multistage decision support. In short, these theories are closer to real life than traditional theories.

The literature on fuzzy decision support is numerous. Related to modular decision support is the application of fuzzy set theory in business, management, or other operational studies. The fuzzy methods belonging to these fields have been deeply developed into various problems, such as optimization problems (linear programming, dynamic programming, etc.), production planning, and similar problems.

Fuzzy set theory has gained more and more attention in the field of computer science, especially for the storage and management of details or information through a way of human thinking, which usually consists of fuzzy details, such as databases, information extraction systems, or expert systems. One of the most prominent advantages of applying fuzzy theory to the above-mentioned systems is that they can use natural language expressions to obtain online information and knowledge and manage them. These advantages make these systems more flexible and realistic. There are quite a lot of relevant literature studies on fuzzy database systems, fuzzy information extraction systems, and fuzzy expert systems.

As to the field of pattern recognition, cluster analysis, and image recognition, fuzzy set theory is also applied quite well. A credible application in these fields is the study of data structures. As can be seen, the purpose of cluster analysis is to try to classify data into several classes (clusters), where the similarity is high in each class, but the interclasses are very low. While in pattern recognition, related features are compared.

Objectively speaking, the application of fuzzy set theory used in engineering fields is more common than in science fields. In terms of engineering fields, the most prominent example is the fuzzy controller. It can be said to be the best proof of application in engineering, and it is commonly cited in general literature. Of course, we cannot mislead others into thinking that there are no other successful applications except for fuzzy controllers. Engineering application was first implied in civil engineering. This is not surprising because in general, every civil engineering project is different, so it relies more on human judgment and general experience than other engineering projects. For example, fuzzy sets have been successfully applied to the evaluation and evaluation of built projects (bridges, highways, buildings, etc.). The objective evaluation of the importance of each component and structure of the building may be conveniently expressed with approximate fuzzy numbers. Then, the overall evaluation of the building can be expressed by the weighted average calculated by the fuzzy algorithm.

Scholars have conducted in-depth research and an application of fuzzy mathematics in many fields. Various industries and fields have been affected by fuzzy mathematics to varying degrees. In practical applications, it is mainly used in engineering technology, pattern recognition, automatic control, artificial intelligence, and decision support, and there are many related papers and works. However, as the social field with most fuzzy phenomena, especially in the field of economic management, the application and research of fuzzy mathematics are relatively weak. In the field of microeconomics and production management, some fuzzy set theories and methods are mainly used to solve problems in management or to analyze certain systems of enterprises in combination with neural networks and artificial intelligence. In general, various fuzzy theories and methods are used to solve various problems in the enterprise [24-26].

In the field of macroeconomics, some scholars have performed some work in this area and put forward their own opinions and opinions about the utilization of fuzzy mathematics to the issues in the field of economics, such as input output, investment analysis, regional economy, etc. For example, some scholars have discussed the basic problems of economics: desire (the integration of the roles of the buyer and seller), satisfaction, and the ambiguity of resources $[27,28]$.

Some scholars have also started with the basic theory of fuzzy sets and described some examples of the application of fuzzy mathematics in economic management, such as the application of fuzzy cluster analysis to assess the level of the enterprise, technology intensity and enterprise benefits, and the use of pattern recognition to determine the operating status of the enterprise. Managers make correct decisions 
and provide correct choices, and use fuzzy comprehensive evaluation models to comprehensively evaluate enterprise ranking, management level, investment projects, and technical and economic issues [29].

In addition, some scholars have analyzed vague phenomena such as the classification of commodities and enterprises from a microperspective, mainly emphasizing the application of cluster analysis in the national economy, while Xu Ruoning, from a statistical perspective, has analyzed economic indicators and regression analysis methods in economics. Fuzzy set theory is applied to revise and improve to make it more in line with economic laws and economic phenomena, thus forming a complete set of fuzzy economic statistical methods and theories. Some scholars have also demonstrated the relationship between fuzzy mathematics, philosophy, and economy in a deep sense, discussed the fuzziness of economics from the perspective of philosophy and mathematics, and used fuzzy theory methods on consumer behavior preferences, business cycles, equality and efficiency, and social welfare. etc., while some scholars discussed and analyzed and proved that using fuzzy set theory in the above-mentioned fields can obtain conclusions that are more consistent with actual economic life. In addition, from the perspective of economic analysis methods, some people have studied fuzzy mathematics by cluster analysis and pattern recognition in forecasting, economic analysis, and inflation. Other scholars have applied fuzzy set theory to study quality economics and enterprise production systems and production plans [30,31].

Therefore, this paper rationally explains the phenomena and laws existing in economic activities from a fuzzy perspective and gives models for fuzzy inference, diagnosis, and production decision-making, which have important meaning and profound theoretical value and practical value.

\section{Fuzzy Decision Theory}

The fuzzy optimization means that after a given set of plans and different issues, seeking a reasonable plan turns into an optimization problem. However, once the objective function or constraint conditions are fuzzy, the issue at this time can be considered as fuzzy optimization. The word "fuzzy" in fuzzy mathematics comes from the English vocabulary Fuzzy, which was originally meant to mean a problem with furry and unclear boundaries. In daily life, ambiguity problems can be seen everywhere. Our human brains have ambiguities in their judgments, such as fat, short; beautiful, ugly; big, small, etc. We unconsciously use fuzzy concepts in our lives. How can we quantify them so that they can be processed quantitatively? In 1965, the American control expert Chad proposed the fuzzy set theory, which marked the birth of fuzzy mathematics. Since then, fuzzy mathematics has caused a worldwide research boom. So far, fuzzy theory has been used in aerospace, railway transportation, national defense and military, and daily electrical appliances. The well-known research theories in this area are as follows: Chad proposed the decomposition theorem and expansion principle, the Japanese Dofu Guanye proposed fuzzy measure and fuzzy integral, and Chad's possibility principle and Simon's satisfactory principle. In my country, fuzzy mathematics has attracted the attention of many of our scholars since the 1980s. For example, Wang Peizhuang put forward the theory of random colony albums, and Chen Shouyu put forward the concept of relative membership degree and established the engineering fuzzy set theory on this basis.

Fuzzy logic theory was founded by the fuzzy set theory, which was proposed by Professor L. A. Zadeh who worked in the Department of Electrical Engineering at the University of California, Berkeley, in 1965. In early times, the fuzzy theory consists of several items, such as fuzzy set theory and fuzzy logic. Besides, it also consists of reasoning and fuzzy control. In addition, it should be mentioned that in the early 1920s, the concept of "vagueness" was proposed by a famous philosopher and mathematician named B. Russell. In his opinion, all the natural languages are vague. For instance, since the concepts of "red" and "old" have no clear connotations and extensions, both of them are unclear and vague. However, in a specific environment, when scholars use these concepts to describe a specific object, they can understand it, which rarely leads to misunderstanding and ambiguity. Professor L.A. Zadeh from the University of California published a famous paper in 1965, in which he first proposed an important concept to express the fuzziness of things: membership function, which broke through Cantor's classical set theory at the end of the $19^{\text {th }}$ century and laid the foundation of fuzzy theory.

In 1974, E.H. Mamdani from Britain realized the world's first experimental steam engine control with fuzzy logic and fuzzy reasoning for the first time and achieved better results than the traditional direct digital control algorit $\mathrm{hm}$, thus announcing the birth of fuzzy control. In 1980, L.P. Holmblad and Ostergard from Denmark adopted fuzzy control in cement kilns and achieved success, which can be considered as the first commercial fuzzy controller with practical significance.

When the random phenomenon is described and solved by the probability and statistics method, the number in the interval $[0,1]$ is used to indicate the probability of things happening with 0,1 , or $d$ indicates whether the event has occurred. The ambiguity is an event with unclear boundaries, which is often a certain degree of occurrence or nonoccurrence. Therefore, when symptoms appear, the number in $[0,1]$ should be used to indicate "whether" it occurs. For those phenomena that have both randomness and ambiguity, the value $r$ in $[0,1]$ can be used to indicate the probability of occurrence, and the value $s$ in $[0,1]$ can be used to indicate the degree of occurrence.

When the index is a fuzzy phenomenon, fuzzy mathematics uses the number in $[0,1]$ to reflect the degree of a certain phenomenon but which value in $[0,1]$ is taken, and different statistical methods will get different levels.

Fuzzy ranking refers to the study of how decision makers determine the order of superiority and inferiority among 
various decision-making schemes in a fuzzy environment. For example, given a fuzzy order (a reflexive, transitive binary fuzzy relationship), or given a nontransitive ordinary binary relationship, how to approximate a total order for multiple indicators and multiple utility functions. The question is how to use the method of fuzzy set theory to synthesize a ranking order, and how to sort the multilevel decision-making problems. These questions have all received preliminary answers.

This kind of promotion in linear programming may result in the study of fuzzy linear programming, and the result is that ordinary linear programming has a wider application range and can adapt to various situations more flexibly. There are two mathematical models in nonlinear programming: asymmetric model and symmetric model.

(1) Asymmetric model: regarding the acceptance of constraints as a prerequisite, the status of the goal and the constraints are not symmetrical. Given the objective function $f(x)$ on the universe $X$ and the fuzzy set $D$ of constraints on $X$, the so-called optimal solution $M$ that maximizes $f$ under the constraint $D$ is a fuzzy subset of $X$, which has membership. When the set at the right end of the equation is an empty set, $\mu M(x)$ is equal to 0 .

(2) Symmetrical model: both the target and the constraint are put in a symmetrical position, given the objective function $f(x)$ and fuzzy restriction set $D$ on the universe $X$.

Let $\mu F(x)=(f(x)-m) /(s-m) F$ be a fuzzy subset of $X$, and its membership function has a linear relationship with the objective function, which is called the target set, denoted as $\mu N(x)=\min (F(x), D(x))(x \in D) ; N$ is the fuzzy optimal solution under the symmetric model.

In summary, scholars have discussed and analyzed the application of fuzzy mathematic theories and methods to problems in economic management from different angles and degrees, and the research in some fields has been quite prominent. Many research studies have been carried out in macro and microeconomics, especially in microlevel applications. Among these, the most widely used method is cluster analysis.

\section{Triangular Fuzzy Number}

A triangular fuzzy number is an important fuzzy number, which has the characteristics of flexible conversion with other fuzzy numbers. In the research of fuzzy set theory based on triangular fuzzy numbers, Yang studied a variety of fuzzy number forms and used them to solve related decision-making problems. Abdel-Basset et al. constructed a new triangular preference relationship based on triangular fuzzy numbers, used consensus to measure the opinions of decision-making experts, and proposed a neutrosophic triangular weighted aggregation operator to solve the fuzzy group decision-making problem. Yang et al. used triangular fuzzy information to propose related operators and applied them to fuzzy multiattribute decision-making problems to solve the problem of online supply chain financial credit risk assessment. Tseng et al. proposed a new interval triangular fuzzy number-grey correlation analysis method to determine the dependent information in the fuzzy system so as to solve the fuzzy multiattribute decision-making problem in the green supply chain. Triangular fuzzy number-related decisionmaking methods can effectively solve problems with uncertainties, and many experts and scholars have also conducted in-depth discussions on them. Amin introduced the triangular neutrophilic cube fuzzy number and proposed the triangular neutrophilic hesitant fuzzy ordered weighted geometric operator to study the multiattribute decision-making method for medical problems. Karimi et al. used triangular fuzzy numbers to improve the best-worst method and verified the effectiveness of the method through an example of the maintenance evaluation of the Boynold District Hospital. In order to reduce the sensitivity of input fuzzy parameters, Seresht proposed a product and norm correlation principle method based on the triangular fuzzy number to perform fuzzy arithmetic operations.

The uncertainty and related indicators in economic management come from two directions: randomness and ambiguity. Randomness is aimed at those who do not know in advance whether they are discovered, but the results are clear after experiments. Tang Wenchao used triangular fuzzy numbers to improve the analytic hierarchy process and demonstrated the rationality of the proposed method through the fault detection rate in the example of the communication system. The TOPSIS method and the VIKOR method are two typical fuzzy multicriteria compromise methods. Professor Opricovic and others expressed the data in the form of triangular fuzzy numbers and compared the VIKOR method with TOPSIS and other decision-making methods from many aspects and pointed out that the VIKOR method can enable decision makers to obtain the optimal compromise solution; thus, the attention of the method is higher and higher. Li Zedong et al. proposed an aggregation method based on the traditional TOPSIS method, combined the TOPSIS method with the entropy weight method for research, and proved the effectiveness of the proposed method through an example of plant drought resistance. Ding Xuefeng and Ma Yu combined the VIKOR method with two-dimensional semantics to solve the fuzzy multicriteria problem based on the ambiguity of language information in the power battery decision-making problem they solved. Liu Zhengmin, Liu Peide, and Liu Weilong proposed an extended multicriteria group decision-making method based on Pythagorean fuzzy sets to evaluate domestic aviation service quality from the perspective of distance measurement.

In this paper, the triangular fuzzy number is expressed as $l(a, b, c)$, which also has the following definition: 


$$
\begin{array}{r}
l(a, b, c)(x)= \begin{cases}0, & x<a, \\
\frac{x-a}{b-a}, & a \leq x \leq b, \\
\frac{c-x}{c-b}, & b<x \leq c, \\
0, & x>c,\end{cases} \\
l(a, a, a)= \begin{cases}1, & x=a, \\
0, & x \neq a,\end{cases} \\
l(0,0, c)(x)= \begin{cases}0, & x \notin(0, c), \\
\frac{c-x}{c}, & x \in(0, c),\end{cases}
\end{array}
$$$$
l(a, 1,1)(x)= \begin{cases}0, & x \notin(a, 1), \\ \frac{x-a}{1-a}, & x \in(a, 1),\end{cases}
$$

where, $a, b$, and $c$ are the real numbers and $a<b<c$. Therefore, the triangular fuzzy number mentioned above is also described in Figure 1.

The following nine types of triangular fuzzy numbers in the interval $[0,1]$ will be used to express weights and evaluation values:

$$
\left\{\begin{array}{l}
n_{1}=l(l 0,0,0.1), \quad n_{2}=l(l 0,0.1,0.3), \quad n_{3}=l(l 0.1,0.3,0.5), \\
n_{4}=l(0.3,0.5,0.7), \quad n_{5}=l(0.5,0.7,0.9), \quad n_{6}=l(0.7,0.9,1), \\
n_{7}=l(0.9,1,1), \quad \overline{1}=l(1,1,1), \quad \overline{0}=l(0,0,0) .
\end{array}\right.
$$

Correspondingly, its special number is shown in Figure 2.

\section{Application of Bidirectional Decision Model in Product Model}

A decision model is a mathematical model used for business decision making. Due to the intricate social and economic system and the crisscrossing of decision-making factors, it may be hard for any decision maker to make the best decision based on intuition and experience alone. Therefore, in modern scientific decision-making, natural science methods and mathematical tools are often used to establish relationship formulas and models between decision-making variables to reflect the essence of decision-making problems and simplify complex decision-making problems. The general form of the decision model is $V=F(A i, S j)$, where $V$ is the value target; $A i$ is the controllable decision-making factor; $S j$ is the uncontrollable decision-making factor. For example, a company has two factories that can both produce A and B products, but the production capacity is different, and the profit is also different. In order for enterprises to have the best economic benefits, they should seek the best production strategy, that is, make a production plan that maximizes the benefits. To this end, the decision model is listed first, and then, the optimal one is obtained through the model. With the development of operation research, effective decision analysis methods such as linear rules, dynamic rules, game theory, queuing theory, deposit and loan models, and scheduling models have emerged. They are all realized by computers and have become practical decision-making methods, that is, mathematical and modeling decision-making methods. Therefore, for more repetitive, including routine management decision making, mathematical models may be utilized to make programs, and computers can be used to achieve automation to realize efficient working. However, it should be mentioned that the solution to a large number of unstructured problems and management decisions cannot be solved by mathematical models, and the important role of people in decision making must be considered, which consists of psychology, social psychology, and behavioral sciences. Therefore, the mathematical model construction is simply a way in the development process of decision science.

Using real numbers on the interval $[0,1]$ will not only lose some useful information but also use the order of real numbers to determine some order of blurring. General language values are used to overcome the shortcomings of real numbers, but it is difficult to apply them in practice due to their complex expressions.

When expressing weight, $n_{1}, \ldots, n_{7}$ denote very low (VL), low (L), medium low (ML), medium (M), medium high (MH), high $(\mathrm{H})$, and very high $(\mathrm{VH})$, respectively. When evaluating influencing factors, $n_{1}, \ldots, n_{7}$ denote very poor $(\mathrm{VP})$, poor $(\mathrm{P})$, medium poor (MP), normal (N), medium good (MG), good $(\mathrm{G})$, and very good (VG), respectively. Of course, 1 and 0 represent absolute high (absolutely good) and absolute low (absolutely bad), respectively.

When evaluating a product, companies can choose some evaluation factor. For example, $C_{1}=$ "Profit," $C_{2}=$ "Repair rate," $C_{3}=$ "Market share," $\ldots C_{n}$. Letting $l_{1}, l_{2}, l_{3}, \ldots, l_{n}$ represent the weight of $C_{1}, C_{2}, C_{3}, \ldots, C_{n}$, while $h_{1}, h_{2}, h_{3}, \ldots, h_{n}$ represent the evaluation of $C_{1}, C_{2}, C_{3}, \ldots, C_{n}$ as follows: 


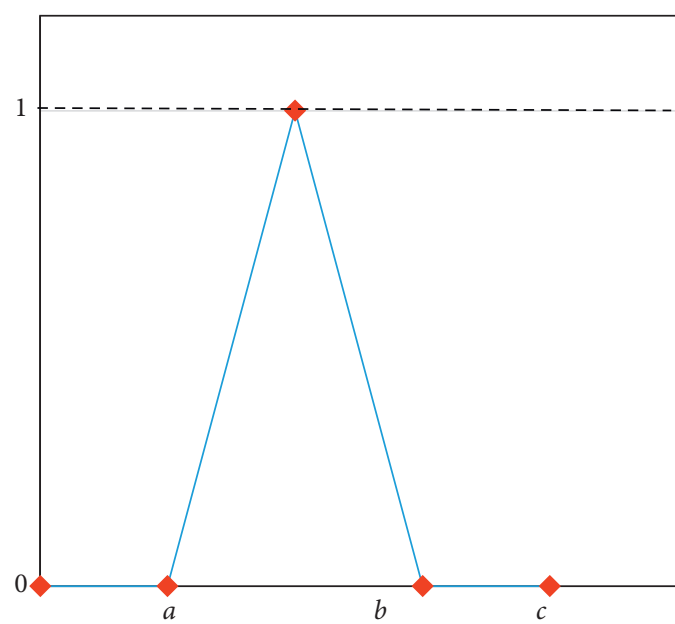

(a)

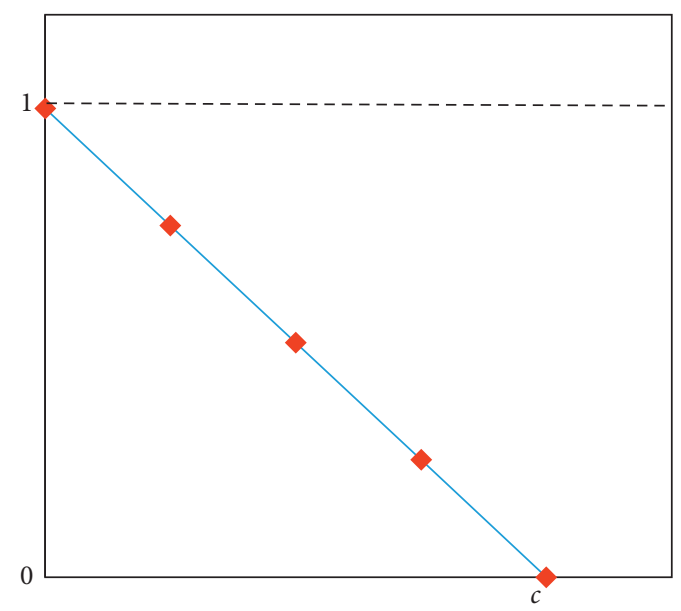

(c)

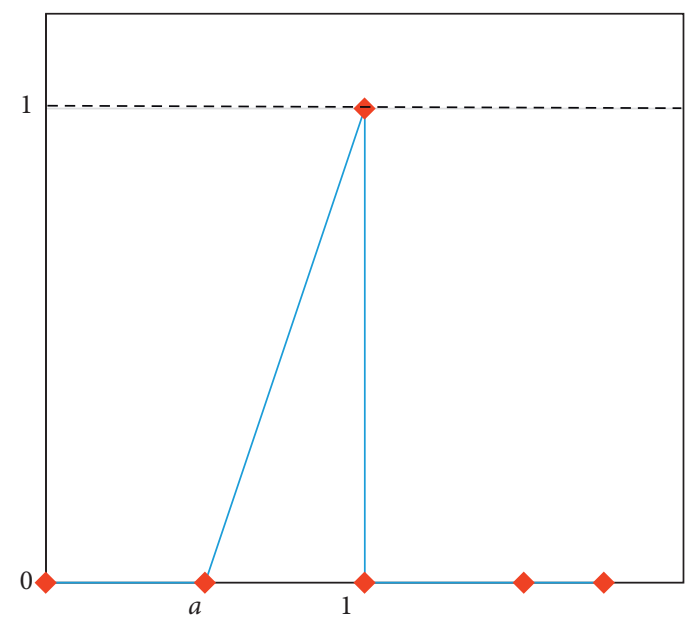

(b)

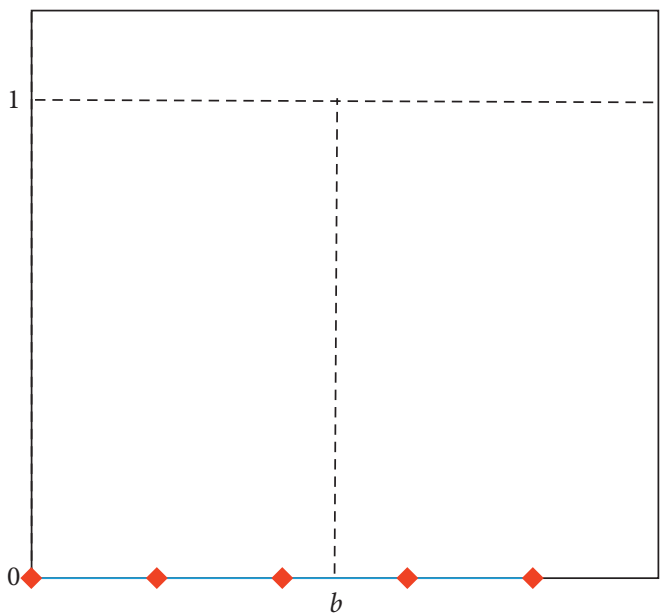

(d)

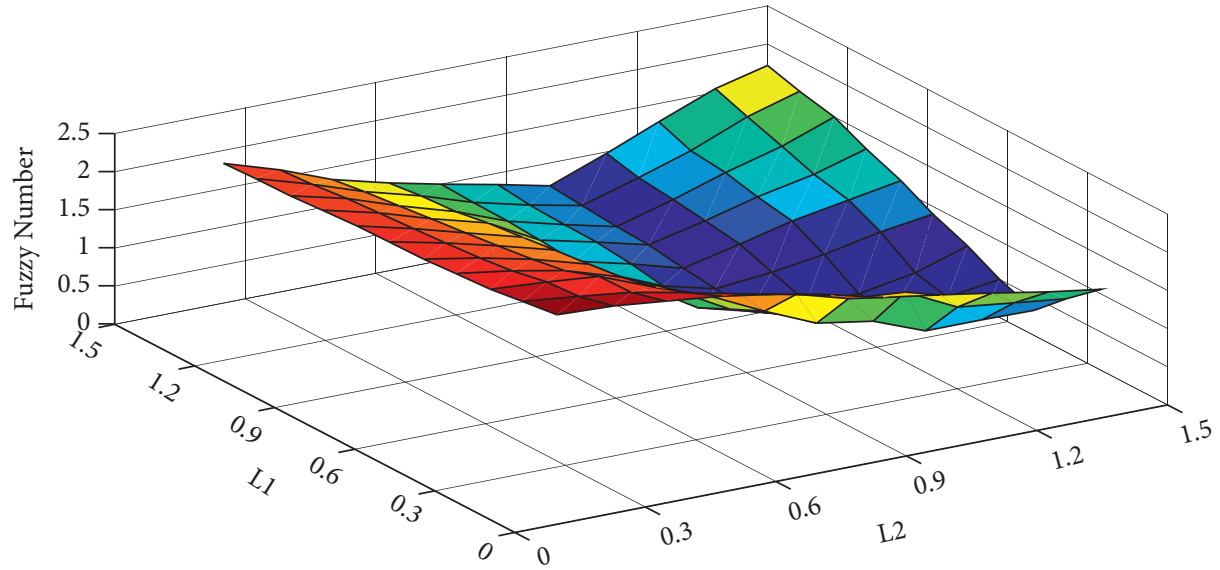

(e)

Figure 1: Triangular fuzzy number. (a) $l(a, b, c)$. (b) $l(a, 1,1)$. (c) $l(0,0, c)$. (d) $l(b, b, b)$. (e) Fuzzy number. 


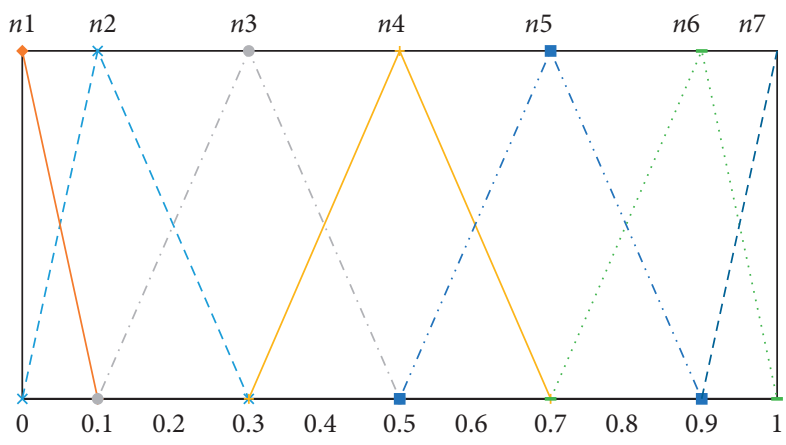

FIgURE 2: Special numbers.

$$
\left\{\begin{array}{l}
l_{k}=l\left(l_{1}^{k}, l_{2}^{k}, l_{3}^{k}\right), \\
h_{k}=l\left(h_{1}^{k}, h_{2}^{k}, h_{3}^{k}\right) .
\end{array}\right.
$$

It can be evaluated separately from two aspects:

$$
\begin{aligned}
A & =\left(l_{1} h_{1}, l_{2} h_{2}, l_{3} h_{3}, \ldots, l_{n} h_{n}\right), \\
a & =l\left(a_{1}, a_{2}, a_{3}\right) .
\end{aligned}
$$

Internal evaluation is mainly inferred from the preferences of consumers. Here, only the public preferences are considered. The influencing factors of consumer decision making may be $D_{1}=$ function, $D_{2}=$ price, $D_{3}=$ appearance, $D_{4}=$ service, $\ldots$, $D_{m}$. The manufacturer can obtain these evaluations using statistical methods, but he cannot obtain accurate weights.

The proposed model is based on the recognition that the level of the potential profitability of producers and the level of internal evaluation of products and operations should be consistent. The basic idea is shown in Figure 3.

\section{The Application of Two-Way Model in Macroeconomics}

The impact of government decisions on social and economic "output" is similar to the impact of producers on their products, and it plays a pivotal and important role. The socio-economic "output" affected by macroeconomic decisionmaking is $C_{1}=\mathrm{GDP}, C_{2}=$ employment, $C_{3}=$ price level, $\ldots, C_{n}$. The injection of government policies into the macroeconomy is like the inputs of producers with many aspects. These "inputs" can be $D_{1}=$ interest rate, $D_{2}=\operatorname{tax}$ rate, $D_{3}=$ labor policy, ..., $D_{m}$.

If $l_{1}, l_{2}, l_{3}, \ldots, l_{n}$ and $h_{1}, h_{2}, h_{3}, \ldots, h_{n}$ are used to represent the weights of $C_{1}, C_{2}, C_{3}, \ldots, C_{n}$ and the evaluation levels of $C_{1}, C_{2}, C_{3}, \ldots, C_{n}$, respectively, the macroeconomic performance evaluation will be simple and can be expressed as

$$
A=\left(l_{1} h_{1}, l_{2} h_{2}, l_{3} h_{3}, \ldots, l_{n} h_{n}\right) .
$$

Obviously, the best and worst evaluations are

$$
1^{*}=(\overline{1}, \overline{1}, \overline{1}, \ldots, \overline{1}) \text { 和 } 0^{*}=(\overline{0}, \overline{0}, \overline{0}, \ldots \overline{0}) \text {. }
$$

The macroeconomic situation is the result of economic activities. These activities are often affected by "internal"

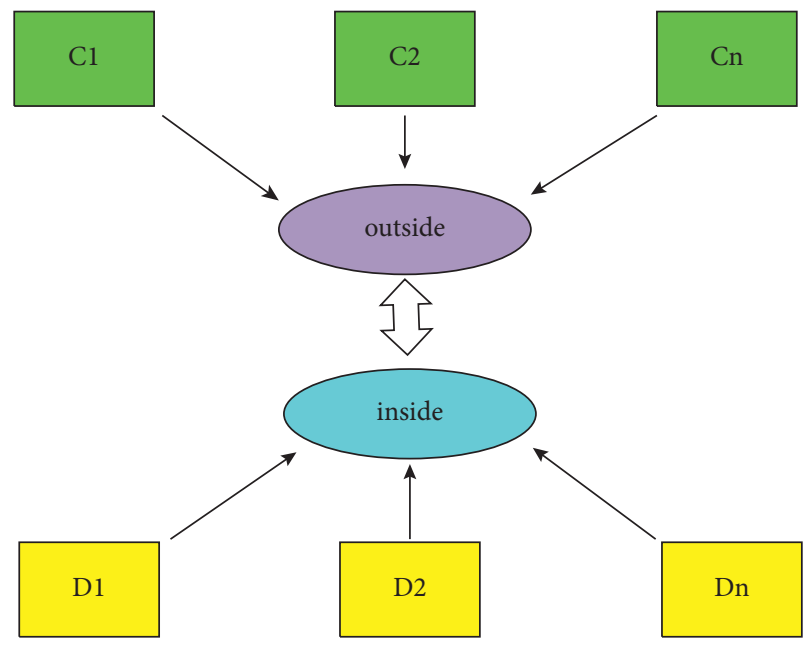

Figure 3: Model structure.

factors " $D_{1}, D_{2}, D_{3}, \ldots, D_{m}$." We establish the following fuzzy sentences similar to economic fuzzy theory.

If each $D_{k}(k=1,2, \ldots, m)$ is appropriate, then the macroeconomic situation will be normal. Let $B^{*}=$ "the macroeconomic situation is appropriate" and $D_{k}=$ " $D_{k}$ is normal" $(k=l, 2, \ldots, m)$, then the above sentence can be expressed as follows:

$$
\left(D_{1}^{*} \wedge D_{2}^{*} \wedge \cdots \wedge D_{m}^{*}\right) \longrightarrow B^{*}
$$

The language value is used to represent the true value of $D_{k}$. Linguistic value is shown in Figure 4 .

Similar to the conversion function in the literature, a function $\Psi_{s}(u)$ can be introduced to convert the value of the degree of reliability, where

$$
\Psi_{s}(u)=u^{1 /(1-s)}, 0 \leq s, u<1 .
$$

Obviously, for each $s$ on $(0,1), \Psi_{s}(u)$ is a 1-1 continuously increasing function of $u$, and for each $u$ on $[0,1]$, the function is a 1-1 continuously decreasing function of $s$. Therefore, the conversion from the external evaluation $\mathrm{A}$ to the $s$ level of the natural language value is given by $\Delta$, $\Psi_{s}(u), \Omega$, and the specific conversion method is shown in Figures 5 and 6 . 

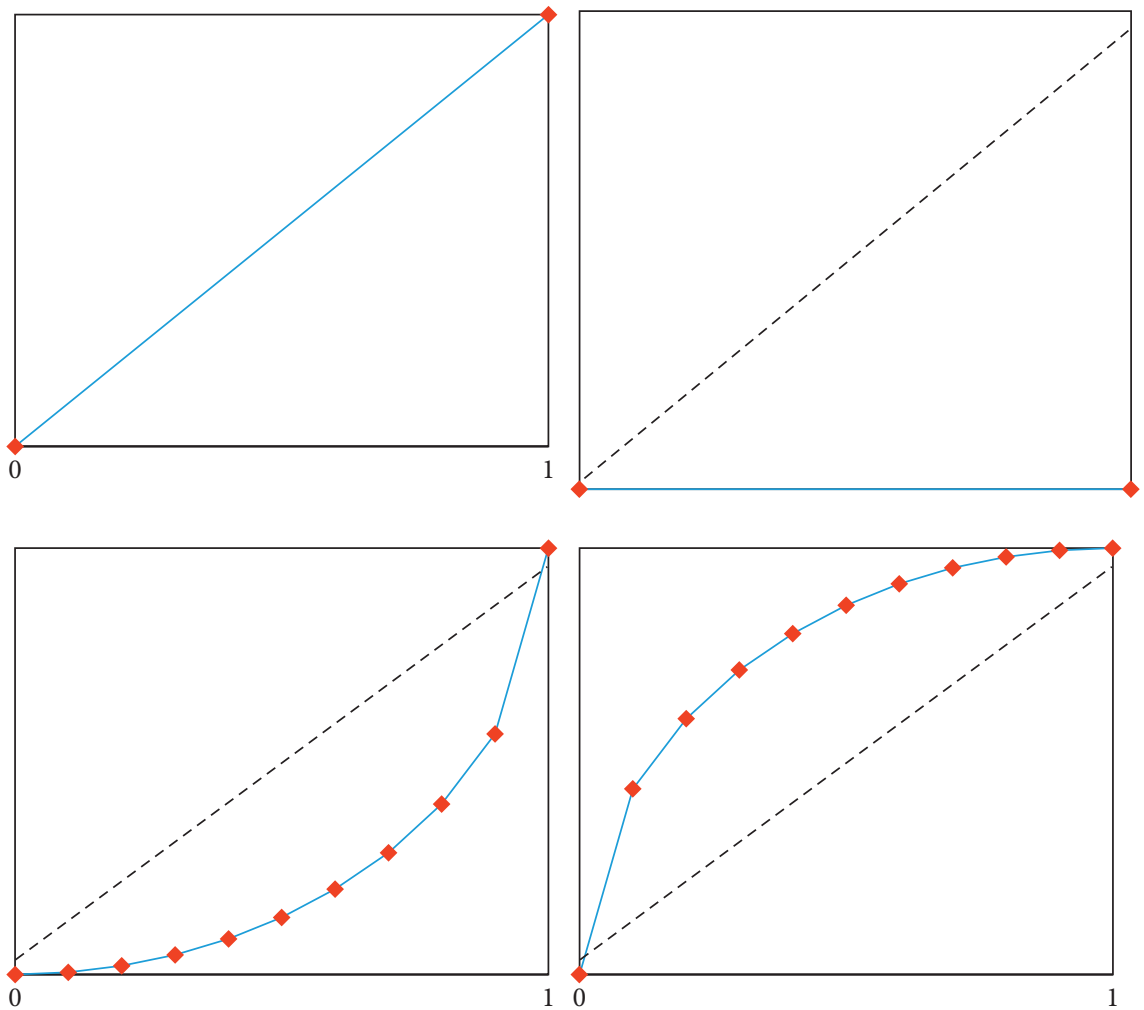

Figure 4: Linguistic value. (a) $t(x)$. (b) $c t(x)$. (c) Very true. (d) Absolutely true.

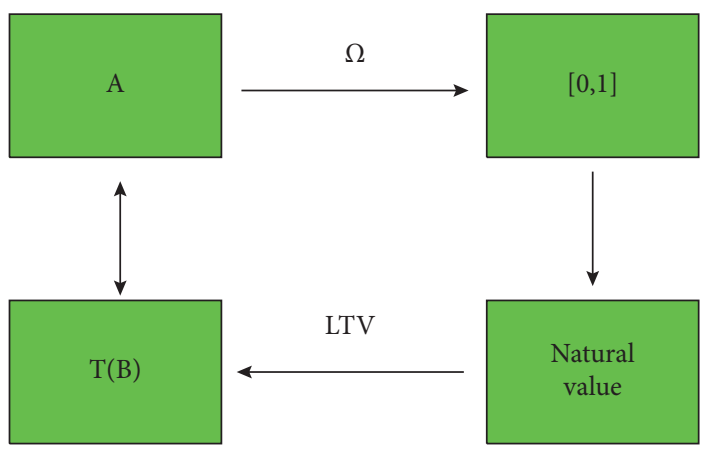

FIGURE 5: Transform.

\section{Example}

Suppose that the manager of a camera company makes decisions about a certain type of product. The internal and external factors are as follows (Tables 1-3): $C_{1}=$ "Profit," $C_{2}=$ "Repair rate," $C_{3}=$ "Market share," $D_{1}=$ "Performance,"
$D_{2}=$ "Price," and $D_{3}=$ "Service." For simplicity, letting $\overline{0}, n_{2}$, $n_{4}, n_{6}$, and $\overline{1}$ denote the weights of $\mathrm{CL}, \mathrm{L}, \mathrm{M}, \mathrm{H}$, and $\mathrm{CH}$, respectively. Besides, let CP, P, UN, G, and CG denote evaluation results. Therefore, Tables $1-3$ are the evaluation results of a group of 3 experts on this issue. Correspondingly, the digital value also can be seen from Figure 7. 


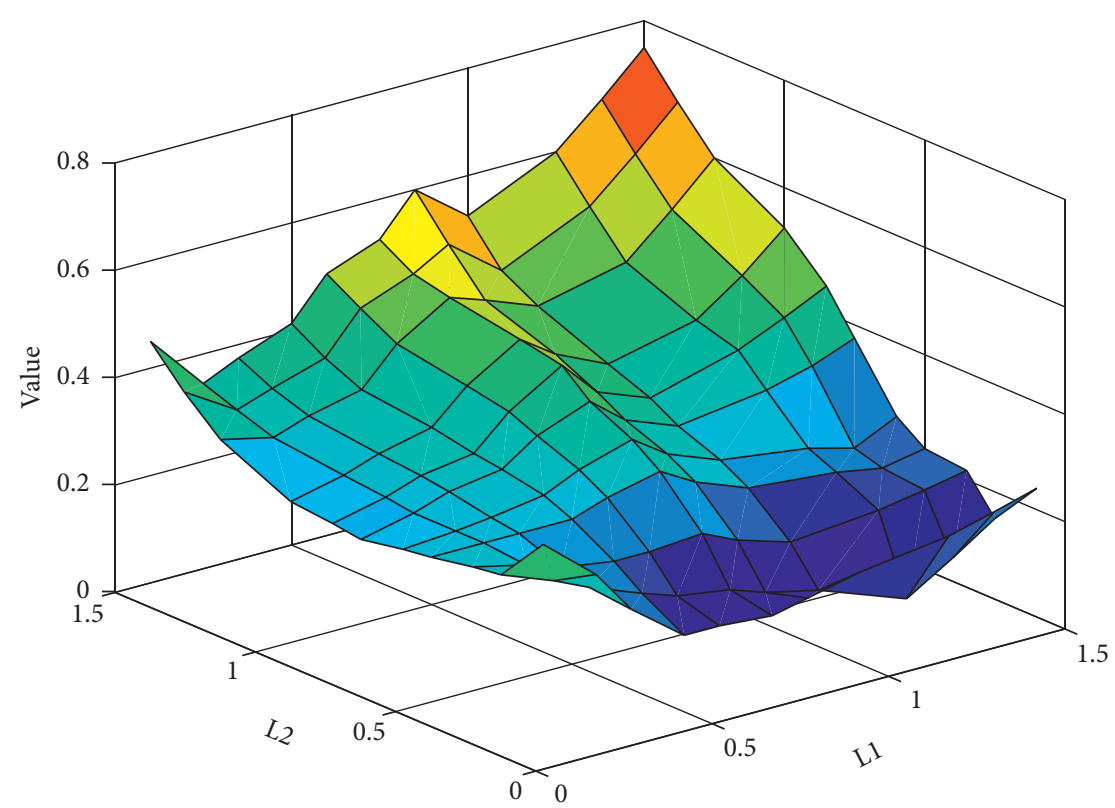

Figure 6: Value vs. $L$.

TABle 1: Weights of C1.

\begin{tabular}{lllll}
\hline & $\mathrm{CL}$ & $\mathrm{L}$ & $\mathrm{M}$ & $\mathrm{H}$ \\
\hline $1^{\text {st }}$ expert & & $\sqrt{ }$ & & \\
$2^{\text {nd }}$ expert & & $\sqrt{ }$ & \\
$3^{\text {rd }}$ expert & & $\sqrt{ }$ & \\
\hline
\end{tabular}

TABLE 2: Evaluation result of C1.

\begin{tabular}{llccc}
\hline & $\mathrm{CP}$ & $\mathrm{P}$ & $\mathrm{UN}$ & $\mathrm{G}$ \\
\hline $1^{\text {st }}$ expert & & $\sqrt{ }$ & \\
$2^{\text {nd }}$ expert & & & $\sqrt{ }$ \\
$3^{\text {rd }}$ expert & & & $\sqrt{ }$ \\
\hline
\end{tabular}

TABLe 3: Evaluation result of D1.

\begin{tabular}{lllll}
\hline & $\mathrm{CP}$ & $\mathrm{P}$ & $\mathrm{UN}$ & $\mathrm{G}$ \\
\hline $1^{\text {st }}$ expert & & & $\mathrm{CG}$ \\
$2^{\text {nd }}$ expert & & $\sqrt{ }$ & \\
$3^{\text {rd }}$ expert & & & $\sqrt{ }$ \\
\hline
\end{tabular}

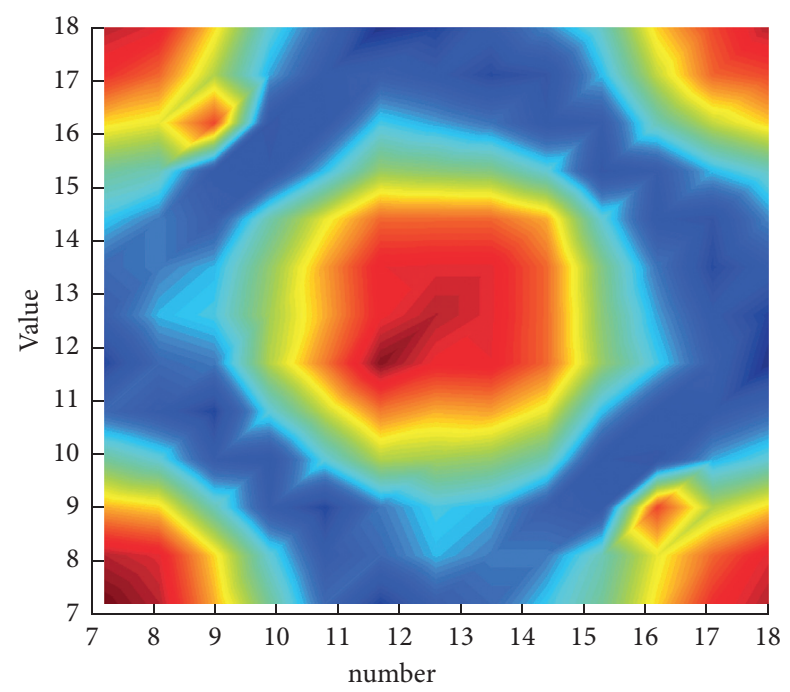

Figure 7: Value vs. number. 


\section{Conclusions}

If users of the model expect to get more information about the "internal" influencing factors, they can do so by adding some evaluation and language values to the model. For example, if we change $b$ to another sentence "the macroeconomic situation is unstable," we can build another model by a similar method. In addition, more useful information can also be obtained from the statistics of production operations and macroeconomics, which will help us to discover more economic relationships in the two-way decisionmaking model.

However, throughout the above writings, domestic and foreign studies have begun on the ambiguities in economic management, especially in the microfields. Although domestic scholars have carried out a lot of research work in the field of economics with the most vagueness, they can find that they have the following problems:

(1) For the vague phenomena in economic management, they simply use fuzzy theory to analyze these phenomena, and this paper explains to simply improve the original traditional methods to make decision-making more reasonable.

(2) In this field, cluster analysis, pattern recognition, and comprehensive evaluation methods are mainly used in the management of microenvironment. There are a very few macroaspects.

(3) Although the fuzzy phenomenon in economic management has been analyzed and researched, it has not revealed the fuzzy correlation of economic phenomena.

(4) How to use fuzzy decision making in the macroeconomic management part of the economic management field is the part that was not involved in the previous work.

\section{Data Availability}

The data used to support the findings of this study are available from the corresponding author upon request.

\section{Conflicts of Interest}

The author declares no conflicts of interest or personal relationships that could have appeared to influence the work reported in this study.

\section{References}

[1] I. Hafeth, A. Naji, and R. Hussein Ali, "Risk response selection in construction projects," Civil Engineering Journal, vol. 3, no. 12, 2017.

[2] Y. Ishida and S. Hashimoto, "Asymmetric characterization of diversity in symmetric stable marriage problems: an example of agent evacuation," Procedia Computer Science, vol. 60, no. 1, pp. 1472-1481, 2015.

[3] D. Gale and L. S. Shapley, "College admissions and the stability of marriage," The American Mathematical Monthly, vol. 69, no. 1, pp. 9-15, 1962.
[4] J. Pais, "Random matching in the college admissions problem," Economic Theory, vol. 35, no. 1, pp. 99-116, 2018.

[5] J. J. Jung and G. S. Jo, "Brokerage between buyer and seller agents using constraint satisfaction problem models," Decision Support Systems, vol. 28, no. 4, pp. 291-384, 2020.

[6] Y. Liu and K. W. Li, "A two-sided matching decision method for supply and demand of technological knowledge," Journal of Knowledge Management, vol. 21, no. 3, pp. 592-606, Article ID JKM-05-2016-0183, 2017.

[7] J. Byun and S. Jang, "Effective destination advertising: matching effect between advertising language and destination type," Tourism Management, vol. 50, no. 10, pp. 31-40, 2015.

[8] D. Gale, "The two-sided matching problem. Origin, development and current issue," International Game Theory Review, vol. 3, no. 2-3, pp. 237-252, 2001.

[9] C. Koch and S. P. Penczynski, “The winner's curse: conditional reasoning and belief formation," Journal of Economic Theory, vol. 174, pp. 57-102, 2018.

[10] C. K. Karl, "Investigating the winner's curse based on decision making in an auction environment," Simulation \& Gaming, vol. 47, no. 3, pp. 324-345, 2016.

[11] D. Ettinger and F. Michelucci, "Creating a winner's curse via jump bids," Review of Economic Design, vol. 20, no. 3, pp. 173-186, 2016.

[12] J. A. Brander and E. J. Egan, "The winner's curse in acquisitions of privately-held firms," The Quarterly Review of Economics and Finance, vol. 65, pp. 249-262, 2017.

[13] Z. Palmowski, "A note on var for the winner's curse," Economics/Ekonomia, vol. 15, no. 3, pp. 124-134, 2017.

[14] B. R. Routledge and S. E. Zin, "Model uncertainty and liquidity," Review of Economic Dynamics, vol. 12, no. 4, pp. 543-566, 2009.

[15] D. Easley and M. O'Hara, “Ambiguity and nonparticipation: the role of regulation," Review of Financial Studies, vol. 22, no. 5, pp. 1817-1843, 2009.

[16] P. Klibano, M. Marinacci, and S. Mukerji, "A smooth model of decision making under ambiguity," Econometrica, vol. 73, no. 6, pp. 1849-1892, 2005.

[17] Y. Halevy, "Ellsberg revisited: an experimental study," Econometrica, vol. 75, no. 2, pp. 503-536, 2007.

[18] C. C. Chow and R. K. Sarin, "Known, unknown, and unknowable uncertainties," Theory and Decision, vol. 52, no. 2, pp. 127-138, 2002.

[19] D. Ahn, S. Choi, D. Gale, and S. Kariv, "Estimating ambiguity aversion in a portfolio choice experiment," Quantitative Economics, vol. 5, no. 2, pp. 195-223, 2009, Working Paper.

[20] T. Hayashi and R. Wada, "Choice with imprecise information: an experimental approach," Theory and Decision, vol. 69, no. 3, pp. 355-373, 2010.

[21] S. Azhar, "Building information modeling (BIM): trends, benefits, risks, and challenges for the AEC industry," Leadership and Management in Engineering, vol. 11, no. 3, pp. 241-252, 2011.

[22] K. Zima, E. Plebankiewicz, and D. Wieczorek, "A SWOT analysis of the use of BIM technology in the polish construction industry," Buildings, vol. 10, no. 1, p. 16, 2020.

[23] P. Sun, B. Liu, and T. Sun, "Injury status and strategies of female 7-a-side rugby players in Anhui Province," Sports Boutique, vol. 38, no. 3, pp. 72-74, 2019.

[24] P. Guild, M. R. Lininger, and M. Warren, "The association between the single leg hop test and lower-extremity injuries in female athletes: a critically appraised topic," Journal of Sport Rehabilitation, vol. 30, no. 2, pp. 320-326, 2020. 
[25] U. G. Inyang, E. E. Akpan, and O. C. Akinyokun, “A hybrid machine learning approach for flood risk assessment and classification," International Journal of Computational Intelligence and Applications, vol. 19, no. 2, Article ID 2050012, 2020.

[26] Q. Liu, S. Du, B. Wyk, and Y. Sun, "Double-layer-clustering differential evolution multimodal optimization by speciation and self-adaptive strategies," Information Sciences, vol. 545, no. 1, pp. 465-486, 2021.

[27] H. R. Medeiros, F. D. Oliveira, H. F. Bassani, and A. Araujo, "Dynamic topology and relevance learning SOM-based algorithm for image clustering tasks," Computer Vision and Image Understanding, vol. 179, pp. 19-30, 2019.

[28] Y. Deng, D. Huang, S. Du, G. Li, and J. Lv, "A double-layer attention based adversarial network for partial transfer learning in machinery fault diagnosis," Computers in Industry, vol. 127, Article ID 103399, 2021.

[29] J. J. Chan, K. K. Chen, S. Sarker et al., "Epidemiology of Achilles tendon injuries in collegiate level athletes in the United States," International Orthopaedics, vol. 44, no. 3, pp. 585-594, 2020.

[30] W. Li, G. G. Wang, and A. H. Gandomi, "A survey of learningbased intelligent optimization algorithms," Archives of Computational Methods in Engineering, vol. 28, pp. 3781-3799, 2021.

[31] G. G. Wang, A. H. Gandomi, A. H. Alavi, and D. Gong, "A comprehensive review of krill herd algorithm: variants, hybrids and applications," Artificial Intelligence Review, vol. 51, no. 1, pp. 119-148, 2019. 